OPEN ACCESS

Edited by:

Guan Zhu,

Texas A\&M University, United States

Reviewed by:

Shuping Zhang,

University of Missouri, United States

Eloiza Teles Caldart,

Universidade Estadual de Londrina

Brazil

*Correspondence:

Qijun Chen

qijunchen759@syau.edu.cn

${ }^{\dagger}$ These authors have contributed equally to this work.

Specialty section:

This article was submitted to Infectious Diseases,

a section of the journal

Frontiers in Microbiology

Received: 15 June 2017 Accepted: 11 October 2017 Published: 26 October 2017

Citation:

Yang $N$, Wang $D$, Xing $M, L i C, L i J$,

Wu A, Sang $X$, Feng $Y$, Jiang $N$ and Chen Q (2017) Seroepidemiology and Risk Factors of Toxoplasma gondii Infection among the Newly Enrolled Undergraduates and Postgraduate Students in China.

Front. Microbiol. 8:2092. doi: 10.3389/fmicb.2017.02092

\section{Seroepidemiology and Risk Factors of Toxoplasma gondii Infection among the Newly Enrolled Undergraduates and Postgraduate Students in China}

\author{
Na Yang', Dawei Wang ${ }^{\dagger}$, Mengen Xing, Chenghuan Li, Jiaqi Li, Anhe Wu, Xiaoyu Sang, \\ Ying Feng, Ning Jiang and Qijun Chen*
}

Key Laboratory of Zoonosis of Liaoning Province, College of Animal Science and Veterinary Medicine, Shenyang Agricultural University, Shenyang, China

Toxoplasma gondii is an obligate intracellular zoonotic parasite, infecting warm-blood animals including humans. Previous serological surveys of $T$. gondii infection have focused on people of different occupations and special groups, such as slaughterhouse workers, AIDS patients and pregnant women. To investigate the potential impact of T. gondii infection on the health of young students, the prevalence of $T$. gondii infection and associated risk factors among the newly enrolled undergraduates and postgraduate students were investigated. A total of 3,569 newly enrolled students (age range: 15- to 37-years-old, median 26 years) from various regions of China were recruited in this study. The serum samples were tested for the presence of $T$. gondii specific IgG by the modified agglutination test (MAT). Questionnaires were used to collect information on risk factors for T. gondii infection. Sixty-five (1.82\%) out of 3,569 participants were seropositive for IgG antibodies to T. gondii by MAT (titer $\geq 1: 20$ ). Four variables were found to be positively associated with $T$. gondii infection, including primary geographical location, living in rural areas, gardening or agriculture, and drinking unboiled water by the univariate logistic regression, and only gardening or agriculture was the independent risk factor for $T$. gondii positivity by using multivariate logistic regression in this study, which may provide information to guide future research and control policies.

Keywords: Toxoplasma gondii, seroepidemiology, risk factors, students, China

\section{INTRODUCTION}

Toxoplasma gondii is an obligate intracellular zoonotic parasite, infecting warm-blood animals, including humans. It has been reported that one-third of the human population worldwide and more than $7 \%$ of the population in China are chronically infected with T. gondii (Dubey, 2010; Zhou et al., 2011; Qin et al., 2014). Humans can be infected through three major ways including consumption of undercooked meat containing T. gondii tissue cysts, ingesting oocystscontaminated water, soil, vegetables and fruits, and transmission from mother to fetus during pregnancy (Dubey, 2010; Wang et al., 2016). 
In women, primary infection during pregnancy can cause severe damage to fetus and newborns including stillbirth, abortion and blindness. It can cause severe infections in individuals with compromised immune systems such as patients with AIDS, cancer treatment or organ transplantation (Dubey, 2010; Zhou et al., 2011). Immune competent individuals infected with $T$. gondii are generally asymptomatic; however, T. gondii infection has been associated with neuropsychiatric disorders suggesting that latent infection may have subtle neurological effects (John et al., 2015).

Toxoplasmosis has become a notifiable disease in China since the first epidemic survey on T. gondii infection in humans was carried out in Guangxi Province in 1978 (Chen et al., 2005). Previous serological surveys of T. gondii infection have focused on people of different occupations, such as slaughterhouse workers, dairy workers, veterinarians, meat-processing workers, cook, and animal breeder, and special groups, such as pregnant women, AIDS and cancer patients, and intravenous drug users (IVDU) in China (Zhou et al., 2011). However, the infection of $T$. gondii in young students and its potential impact has not been investigated. In this study, the prevalence of $T$. gondii infection and associated risk factors among the newly enrolled 3,569 undergraduates and postgraduate students of Shenyang Agricultural University from all over China was investigated.

\section{MATERIALS AND METHODS}

\section{Participants}

A total of 3,569 whole blood samples of newly enrolled undergraduates and postgraduate students of Shenyang Agricultural University originated from various regions of China were collected in September 2016 to survey the presence of T. gondii specific antibodies. The-original regions of the students are mainly located in eastern, southern, central, north, northwest, southwestern, and northeastern regions of China. The age of the newly enrolled students ranged from 15 to 37 years (median 26 years).

\section{Ethics Statement}

This study was carried out in accordance with the recommendations of the Guidelines for Using Subjects from Humans and Animals, Ethical Committee of Shenyang Agricultural University (Clearance number 2015-CAV-01) with written informed consent from all participants. All subjects gave written informed consent in accordance with the Declaration of Helsinki.

\section{Serological Testing}

The sera of all participants were tested for the specific IgG to T. gondii by the modified agglutination test (MAT) as described by Dubey and Desmonts (1987) and the T. gondii Whole Cell Antigen was purchased from Kerafast, Inc. (Boston, MA, United States). MAT has been regarded as the gold-standard for T. gondii infection test. Twofold dilutions of the sera were performed from 1:20 to 1:1,280. The test was regarded positive when a layer of agglutinated parasite antigen was formed in wells at dilutions of 1:20 or higher; positive and negative controls were included in each test. Each serum was tested for three times.

\section{Questionnaire}

The questionnaire contained information of basic demographic data, including age, gender, education background and residence before university. Possible risk factors, including drinking unboiled water, raw or not well-cooked meat (including lamb, beef, pork, fish, oyster) and raw vegetable consumption, animal contacts (cats or dogs), gardening or agricultural activities, blood transfusion and living in urban areas or countryside.

\section{Statistical Analysis}

For the statistical analysis, the SPSS 13.0 software package (IBM, Armonk, NY, United States) was used. $P$-values less than 0.05 were considered statistically significant. Logistic regression was used to analyze the association between $T$. gondii infection and potential risk factors. Multivariate logistic analysis was further performed with the full model, including all potential risk factors in the analyses.

\section{RESULTS}

\section{Seroprevalence of $T$. gondii Infection}

In the present study, $65(1.82 \%)$ of the 3,569 participants were seropositive for anti-T. gondii IgG by MAT, with titers of 1:20 in $35,1: 40$ in 20, and 1:80 in 10 participants. The seroprevalence of T. gondii infection in postgraduate students $(2.46 \%)$ was higher than that in undergraduates (1.63\%), however, the difference was not statistically significant $(p>0.05)$ (Tables 1,2$)$. By gender, the anti-T. gondii IgG seropositive rates was $1.91 \%(26 / 1360)$ in men and $1.31 \%(29 / 2209)$ in women $(p=0.751)$, respectively (Tables 1, 2).

By geographic region, seroprevalence of $T$. gondii infection was $1.49 \%(5 / 335)$ in Eastern China, 5.55\% (2/36) in Southern China, 2.15\% (6/279) in Central China, $1.37 \%(6 / 439)$ in Northern China, $1.97 \%$ (3/152) in Northwest China, 6.58\% $(15 / 228)$ in Southwest China, $1.34 \%(28 / 2,076)$ in Northeast China (Figure 1 and Table 1). In addition, the seroprevalence of $T$. gondii infection varied in different geographical regions, ranging from 1.44 to $4.62 \%$, with a general tendency of a lower prevalence in the North and higher in the South (Tables 2, 3). Students originated from provinces of Hainan and Chongqing showed the significantly higher infection rates (11.11 and $15.38 \%$, respectively).

The participants were also allocated to 3 age groups: 16to 19-years-old, 20- to 25-years-old, and 26- to 37-years-old. The seroprevalence of $T$. gondii infection varied in different age groups, ranging from 1.57 to $3.52 \%$, with a general tendency for older participants to have a higher prevalence, compared with younger participants (Table 1).

\section{Risk Factors for T. gondii Infection}

In the univariate analysis, four variables were found to be associated with anti-T. gondii IgG positivity, including geographical location, living in rural areas, gardening or 
TABLE 1 | Demographic characteristics and seroprevalence of Toxoplasma gondii infection in 3,569 participants tested by the modified agglutination test (MAT).

\begin{tabular}{|c|c|c|c|c|}
\hline Characteristics & & Samples & No. positive & Positive rate (\%) \\
\hline & South China & 36 & 2 & 5.55 \\
\hline & North China & 439 & 6 & 1.37 \\
\hline & Northwest China & 152 & 3 & 1.97 \\
\hline & Southwest china & 228 & 15 & 6.58 \\
\hline & Unknown regions & 24 & 0 & 0 \\
\hline \multirow[t]{2}{*}{ Gender } & Male & 1,360 & 26 & 1.91 \\
\hline & Female & 2,209 & 29 & 1.31 \\
\hline \multirow[t]{3}{*}{ Age } & $16-19$ & 2,362 & 37 & 1.57 \\
\hline & $20-25$ & 1,122 & 25 & 2.22 \\
\hline & $26-37$ & 85 & 3 & 3.52 \\
\hline \multirow{4}{*}{ Blood group type } & $B$ & 398 & 4 & 1.01 \\
\hline & $A B$ & 155 & 1 & 0.64 \\
\hline & $\mathrm{O}$ & 437 & 8 & 1.83 \\
\hline & Unknown & 2,275 & 42 & 1.84 \\
\hline Total & & 3,569 & 65 & 1.82 \\
\hline
\end{tabular}

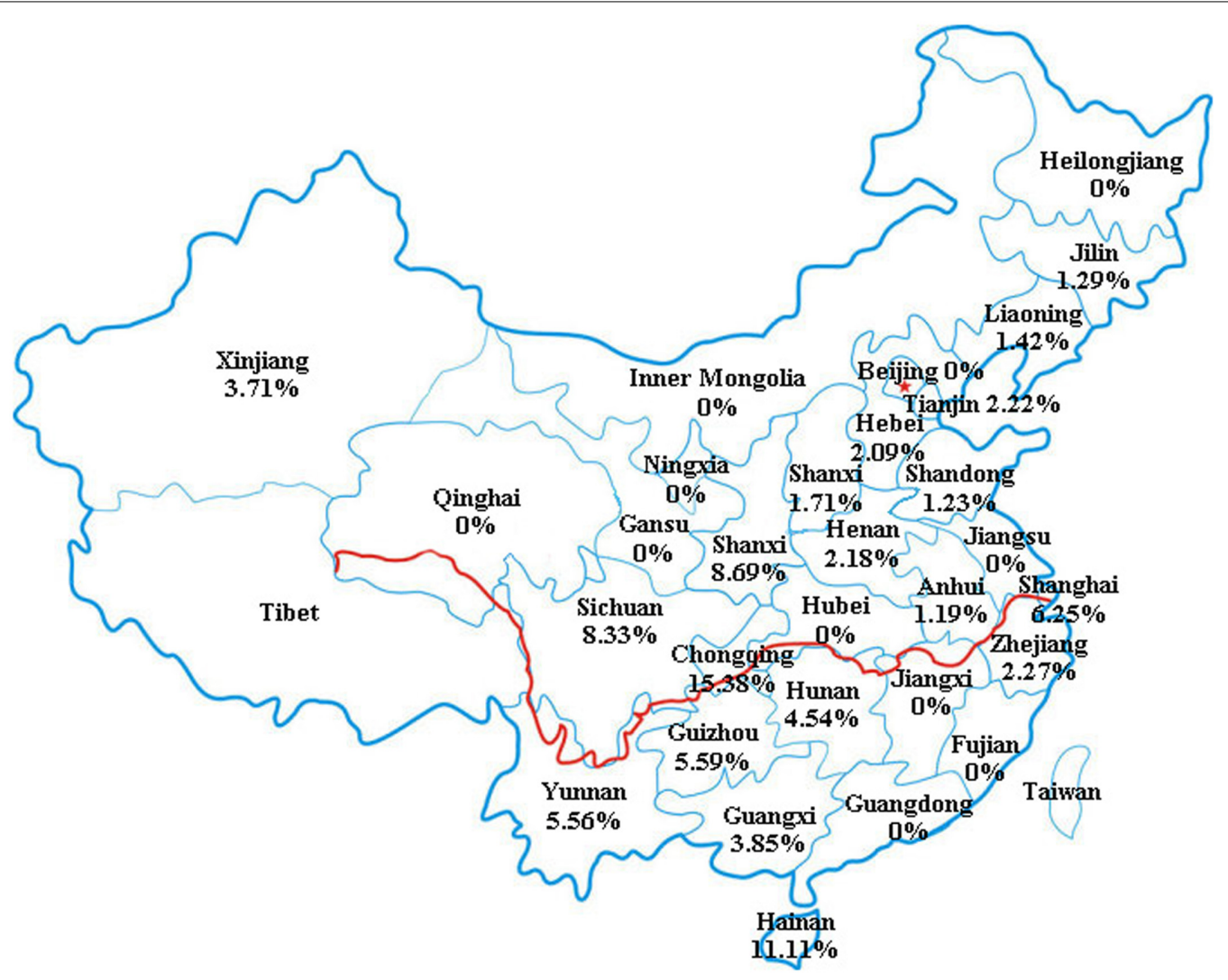

FIGURE 1 | Geographical locations where this study was carried out. 
TABLE 2 | Univariate analysis of the variables associated with $T$. gondii seroprevalence in participants tested by MAT.

\begin{tabular}{|c|c|c|c|c|}
\hline Variable & Blood donor No. & Seropositivity (\%) & Odds ratio ( $95 \%$ Confidence internal) & $P$-value \\
\hline \multicolumn{5}{|l|}{ Geographical location } \\
\hline Unknown & 24 & 0 & 0.000 & 0.998 \\
\hline South of the Yangtze River & 303 & 4.62 & $1.3(0.5-3.3)$ & 0.562 \\
\hline Yangtze River Province & 197 & 3.55 & 1 & \\
\hline \multicolumn{5}{|l|}{ Living in rural areas or city } \\
\hline Rural areas & 1,386 & 2.59 & 1 & \\
\hline \multicolumn{5}{|l|}{ Cat in the household } \\
\hline Yes & 299 & 1.67 & 0.000 & 0.998 \\
\hline No & 3,270 & 1.83 & 1 & \\
\hline \multicolumn{5}{|l|}{ Cat in the neighborhood } \\
\hline Yes & 37 & 0 & $0.9(0.4-2.3)$ & 0.841 \\
\hline No & 2,796 & 2.01 & 1 & \\
\hline \multicolumn{5}{|l|}{ Drink unboiled water } \\
\hline Yes & 1,340 & 2.46 & $1.7(1.1-2.8)$ & $0.028^{*}$ \\
\hline No & 2,229 & 1.43 & 1 & \\
\hline \multicolumn{5}{|c|}{ Undercooked beef meat/lamb consumption } \\
\hline Yes & 240 & 1.25 & $0.7(0.2-2.1)$ & 0.496 \\
\hline No & 3,329 & 1.86 & 1 & \\
\hline \multicolumn{5}{|c|}{ Undercooked pork meat consumption } \\
\hline Yes & 46 & 0 & 0.000 & 0.998 \\
\hline No & 3,523 & 1.85 & 1 & \\
\hline \multicolumn{5}{|l|}{ Raw fish consumption } \\
\hline No & 1,371 & 2.26 & 1 & \\
\hline \multicolumn{5}{|l|}{ Gardening or agriculture } \\
\hline Yes & 644 & 4.04 & $3.1(1.9-5.2)$ & $0.000^{*}$ \\
\hline No & 2,925 & 1.33 & 1 & \\
\hline \multicolumn{5}{|l|}{ Blood transfusion } \\
\hline Yes & 41 & 0 & 0.000 & 0.998 \\
\hline No & 3,528 & 1.85 & 1 & \\
\hline \multicolumn{5}{|l|}{ Blood group type } \\
\hline Unknown & 2,275 & 1.84 & $2.9(0.4-21.2)$ & 0.295 \\
\hline $\mathrm{O}$ & 437 & 1.83 & $2.9(0.4-23.1)$ & 0.322 \\
\hline A & 304 & 3.29 & $5.2(0.7-41.3)$ & 0.116 \\
\hline$B$ & 398 & 1.01 & $1.6(0.2-14.1)$ & 0.690 \\
\hline$A B$ & 155 & 0.64 & 1 & \\
\hline \multicolumn{5}{|l|}{ Gender } \\
\hline Male & 1,360 & 1.91 & $1.1(0.7-1.8)$ & 0.751 \\
\hline Female & 2,209 & 1.31 & 1 & \\
\hline \multicolumn{5}{|l|}{ Education level } \\
\hline Undergraduate & 2,756 & 1.63 & $0.7(0.4-1.1)$ & 0.124 \\
\hline Postgraduate students & 813 & 2.46 & 1 & \\
\hline
\end{tabular}

*Statistically significant. 
TABLE 3 | The seroprevalence of T. gondii infection in 3,569 participants from different geographical regions in China.

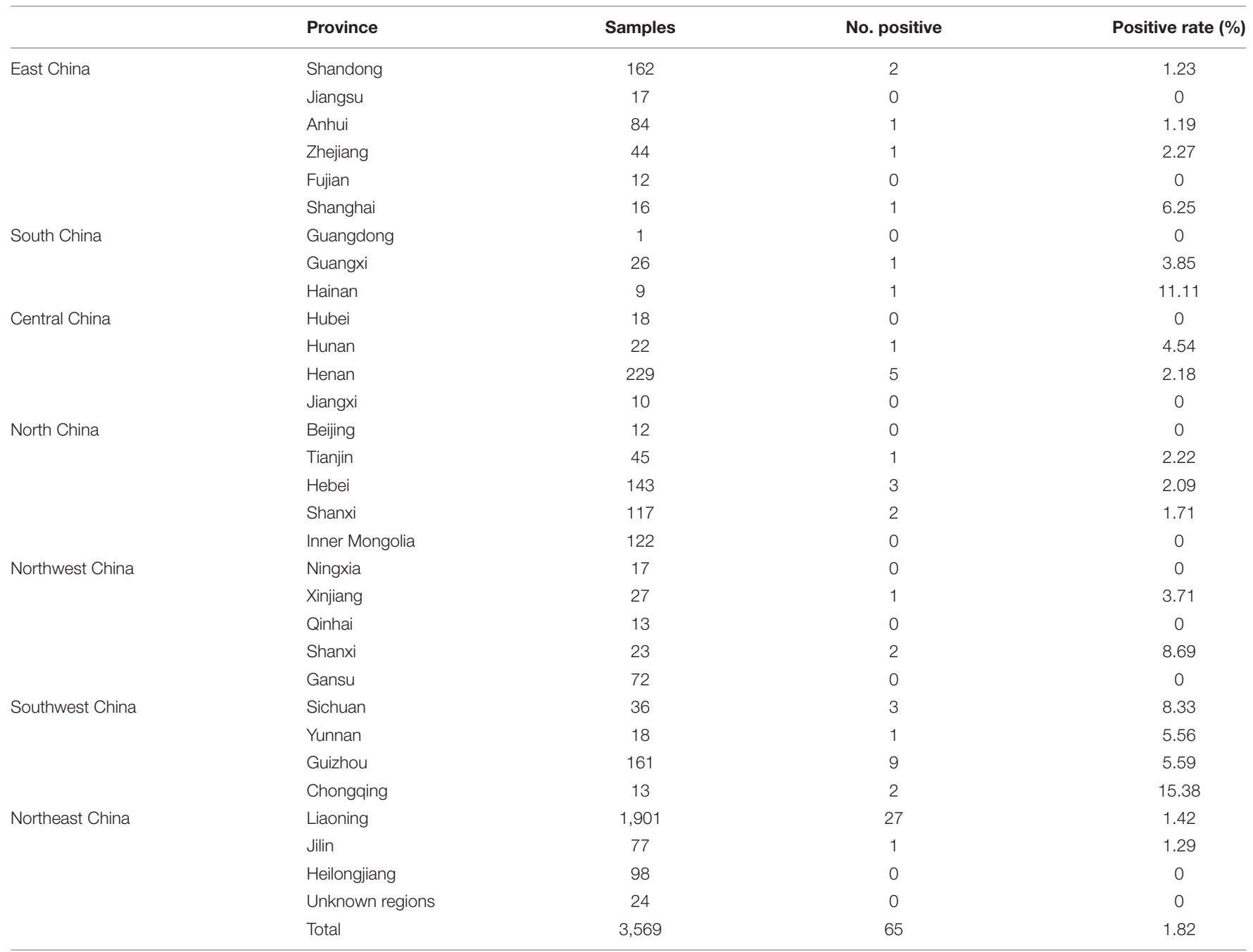

agriculture, and drinking unboiled water (Table 2). Further analysis using multivariate logistic regression revealed that gardening or agriculture was independent risk factor for $T$. gondii seropositivity (Table 4).

\section{DISCUSSION}

In this study, $T$. gondii infection among newly enrolled undergraduates and postgraduate students were systematically investigated. The overall prevalence of $T$. gondii antibodies among these participants was $1.82 \%(65 / 3,569)$; the infection rates of the students coming from eastern, southern, central, north, northwest, southwestern and northeastern regions of China are $1.49,5.55,2.15,1.37,1.97,6.58$, and $1.34 \%$, respectively. These results indicated that $T$. gondii infection occurred earlier in students in all geographical regions in China, but at lower infection rate than that of total population reported earlier (Xiao et al., 2010; Chiang et al., 2012; Yang et al., 2013). The differences between the current study and the previous reports were likely due to the study population. Studies earlier focused predominantly on adult groups with different professions and disease exposures or co-infection (Zhou et al., 2011). Here, we only focused young students before university. It was reported that the seroprevalence of $T$. gondii infection in students was 3.2\% in São Paulo State University (UNESP), in Assis, São Paulo state, Brazil (Rodrigues et al., 2015), which was higher than the infection rate in this study. Moreover, the seroprevalence of T. gondii infection varied in different geographical areas with a general tendency of lower infection rate in the Northern region of Yangtze River (1.44\%) and higher infection rate in the Southern region of Yangtze River (4.62\%) (Table 1). However, the infection rates in Hainan, Sichuan and Chongqing are the highest (Table 3). The causes for these variations in infection rates in the areas are not yet known. Environmental conditions and regional climate may determine the degree of natural distribution of $T$. gondii infection. It has been reported that infection is more prevalent in warm climates and high rain fall areas than in cold climates and dry areas (Dubey, 2010). For the three provinces with the highest infection rates, the habit of food consumption 
TABLE 4 | Multivariate logistic regression with full model for risk factors of T. gondii infection.

\begin{tabular}{|c|c|c|}
\hline Variable & Odds ratio ( $95 \%$ Confidence internal) & $P$-value \\
\hline Geographical position (unknown vs. Yangtze River Province) & 0.0 & 0.998 \\
\hline Geographical position (North of the Yangtze River vs. Yangtze River Province) & $0.4(0.2-1.0)$ & 0.050 \\
\hline Geographical position (South of the Yangtze River vs. Yangtze River Province) & $1.2(0.4-3.0)$ & 0.748 \\
\hline Living in rural areas or city & $0.8(0.4-1.6)$ & 0.620 \\
\hline Cat in the household & 0.0 & 0.998 \\
\hline Cat in the neighborhood & $0.8(0.3-2.2)$ & 0.728 \\
\hline Keep a dog & $0.4(0.2-0.9)$ & 0.021 \\
\hline Drinking unboiled water & $1.6(0.9-2.8)$ & 0.084 \\
\hline Undercooked beef meat/lamb consumption & $0.9(0.3-3.2)$ & 0.889 \\
\hline Undercooked pork meat consumption & 0.0 & 0.998 \\
\hline Raw fish consumption & $1.1(0.5-2.6)$ & 0.845 \\
\hline Fresh oyster consumption & $2.0(0.9-4.4)$ & 0.087 \\
\hline Undercooked vegetables consumption & $0.7(0.4-1.2)$ & 0.173 \\
\hline Gardening or agriculture & $3.0(1.5-5.7)$ & 0.001 \\
\hline Blood transfusion & 0.0 & 0.998 \\
\hline Blood group type (Unknown vs. AB) & $2.4(0.3-17.5)$ & 0.403 \\
\hline Blood group type (O vs. AB) & $2.8(0.3-22.9)$ & 0.334 \\
\hline Blood group type (A vs. AB) & $4.7(0.6-37.8)$ & 0.143 \\
\hline Blood group type (B vs. AB) & $1.5(0.2-13.3)$ & 0.740 \\
\hline Gender & $0.8(0.5-1.4)$ & 0.522 \\
\hline Education level & $0.6(0.3-1.0)$ & 0.058 \\
\hline
\end{tabular}

may be the main reason. People in Hainan Province consume more sea food than other areas and instant-boiling meat is very popular in Chongqing and Sichuan areas. Further, environmental conditions favoring sporulation and survival of oocysts may be another reason for the high prevalence in these regions. Rainfalls in Southern China are much heavier than that in the North, which facilitate and oocysts survived outdoors in soil will be brought to the surface and spread to other places to expand the scope of $T$. gondii infection. In addition, it is likely that oocysts are easily carried into homes on shoes in rainy and humid conditions (Kniel et al., 2002; Dubey, 2010). Moreover, drying under low humidity in the North of the Yangtze River were deleterious to oocysts (Dubey, 2010).

In this study, drinking unboiled water is found to be a significant risk factor for $T$. gondii seropositivity among these participants $(p=0.028$, adjusted OR $=3.1, \mathrm{CI}: 1.9-5.2)$ (Table 2). This result indicated that contamination of drinking water by $T$. gondii are common, and drinking boiled water is a way of avoiding $T$. gondii infection in China. The result also speaks for the importance of the surveillance of water hygiene.

Among the participants in this study, both living in rural areas and gardening or agriculture are also significant risk factors for T. gondii infection, which was similar to the previous study (Mahmoudvand et al., 2015). The prevalence of T. gondii seropositivity was significantly higher in the participants living in rural areas (2.59\%) than those living in cities $(1.33 \%)(p=0.009$; adjusted OR $=0.5 ; 95 \%$ CI: $0.3-0.8$ ) (Table 2 ). Individuals with frequent gardening and farming activities are more easily exposure to T. gondii ( $p=0.000$; adjusted OR $=3.1 ; 95 \%$ CI: 1.9-5.2) (Table 2), which were independent risk factors for T. gondii seropositivity by multivariate logistic regression analysis
( $p=0.001$; adjusted OR $=3.0 ; 95 \%$ CI: $1.5-5.7$ ) (Table 4). These results indicated that people living in rural areas or farming had more chance to contact with oocysts shed in the feces of infected cats. The finding that $T$. gondii infection rate of ranging chicken was much higher than that of caged chicken also support our data (Xiao et al., 2010).

Moreover, we found that fresh oyster consumption was another potential risk factor for $T$. gondii infection though the difference was not statistically significant (Tables 2, 4), which was similar to the study from the United States (Jones et al., 2009). It was reported that $T$. gondii oocysts could be washed into the sea via runoff (Lindsay et al., 2003; Dubey, 2004), and oysters, clams, and mussels could ingest oocysts directly from seawater (Lindsay et al., 2003; Dubey, 2004, 2010). In China, fresh oyster has become more popular in recent years, this may explain the higher infection rate in the participants originated from Hainan Province.

In this study, the seroprevalence of $T$. gondii infection showed a general tendency with aging. Meanwhile, seroprevalence of $T$. gondii infection in postgraduate students $(2.46 \%)(\geq 22$-yearsold) was higher than that in undergraduates $(1.63 \%)(\leq 21$ years-old). These results suggested that older participants may have more chance of $T$. gondii infection, though there was no statistical significance between the two groups. This is in agreement with other reports (Agmas et al., 2015; Cong et al., 2015). In addition, the anti-T. gondii IgG-seropositive rates was $1.91 \%(26 / 1,360)$ and $1.31 \%(29 / 2,209)$ in men and women ( $p=0.751)$, respectively, contrary to the previous report (Zemene et al., 2012). This might be due to the living conditions which are very different between the countries.

Cats are the main definite host of T. gondii, and oocysts are shed from infected cat feces constantly, which are a major source 
of T. gondii infection in humans (Dubey, 2010). Contact with cats is consistently demonstrated to be a risk factor in prior seroepidemiologic studies (Chiang et al., 2012; Zemene et al., 2012; Agmas et al., 2015; Cong et al., 2015; Rodrigues et al., 2015). However, this study did not show any association between $T$. gondii infection with keeping cats $(p>0.5)$. According to the questionnaire survey, although these students once had cats, they rarely contacted with the cats because of perennial learning in school.

\section{CONCLUSION}

This study has shown that the general infection rate of T. gondii among the newly enrolled undergraduates and postgraduate students in China was $1.82 \%$ which is much lower than that obtained from the total population. Four variables were found to be associated with $T$. gondii infection, including geographical location, living in rural areas, gardening or agriculture, and drinking unboiled water by the univariate logistic regression, and

\section{REFERENCES}

Agmas, B., Reta Tesfaye, R., and Koye, D. N. (2015). Seroprevalence of Toxoplasma gondii infection and associated risk factors among pregnant women in Debre Tabor, Northwest Ethiopia. BMC Res. Notes 8:107. doi: 10.1186/s13104-0151083-2

Chen, X. G., Wu, K., and Lun, Z. R. (2005). Toxoplasmosis researches in China. Chin. Med. J. 118, 1015-1021.

Chiang, T. Y., Hsieh, H. H., Kuo, M. C., Chiu, K. T., Lin, W. C., Fan, C. K., et al. (2012). Seroepidemiology of Toxoplasma gondii infection among healthy blood donors in Taiwan. PLOS ONE 7:e48139. doi: 10.1371/journal.pone.0048139

Cong, W., Dong, W., Bai, L., Wang, X. Y., Ni, X. T., Qian, A. D., et al. (2015). Seroprevalence and associated risk factors of Toxoplasma gondii infection in psychiatric patients: a case-control study in eastern China. Epidemiol. Infect. 143, 3103-3109. doi: 10.1017/S0950268814003835

Dubey, J. P. (2004). Toxoplasmosis - a waterborne zoonosis. Vet. Parasitol. 126, $57-72$.

Dubey, J. P. (2010). Toxoplasmosis of Animals and Humans, 2nd Edn. (Boca Raton, FL: CRC Press), 1-313.

Dubey, J. P., and Desmonts, G. (1987). Serological responses of equids fed Toxoplasma gondii oocysts. Equine Vet. J. 19, 337-339.

John, C. C., Carabin, H., Montano, S. M., Bangirana, P., Zunt, J. R., and Peterson, P. K. (2015). Global research priorities for infections that affect the nervous system. Nature 527, S178-S186. doi: 10.1038/nature16033

Jones, J. L., Dargelas, V., Roberts, J., Press, C., Remington, J. S., and Montoya, J. G. (2009). Risk factors for Toxoplasma gondii infection in the United States. Clin. Infect. Dis. 49, 878-884. doi: 10.1086/605433

Kniel, K. E., Lindsay, D. S., Sumner, S. S., Hackney, C. R., Pierson, M. D., and Dubey, J. P. (2002). Examination of attachment and survival of Toxoplasma gondii oocysts on raspberries and blueberries. J. Parasitol. 88, 790-793.

Lindsay, D. S., Collins, M. V., Mitchell, S. M., Cole, R., Flick, G., Wetch, C. N., et al. (2003). Sporulation and survival of Toxoplasma gondii oocysts in sea water. J. Eukaryot. Microbiol. 50, S687-S688.

Mahmoudvand, H., Saedidezaki, E., Soleimani, S., Baneshi, M. R., Kheirandish, F., Ezatpour, B., et al. (2015). Seroprevalence and risk factors of Toxoplasma gondii infection among healthy blood donors in south-east of Iran. Parasite Immunol. 37, 362-367. doi: 10.1111/pim.12198 only gardening or agriculture was the independent risk factor for T. gondii seropositivity by using multivariate logistic regression in this study.

\section{AUTHOR CONTRIBUTIONS}

NY and QC conceived and designed the study. NY, DW, MX, $\mathrm{CL}$, JL, and AW performed the study. XS, YF, and NJ helped to collected the samples. NY and QC wrote the manuscript.

\section{FUNDING}

This work was supported by the National Key R\&D Program of China [2017YFD0500400], the National Natural Science Foundation of China [grant no. NSFC 31201894, 31672546], China Postdoctoral Science Foundation [grant no. 2014M561252], and Tianzhu mountain talent program of Shenyang Agricultural University.

Qin, S. Y., Cong, W., Liu, Y., Li, N., Wang, Z. D., Zhang, F. K., et al. (2014). Molecular detection and genotypic characterization of Toxoplasma gondii infection in bats in four provinces of China. Parasit. Vectors 7:558. doi: 10.1186/s13071-014-0558-7

Rodrigues, J. P., Frei, F., Navarro, I. T., Silva, L. P., Marcelino, M. Y., AndradeJunior, H. F., et al. (2015). Seroepidemiological analysis of toxoplasmosis in college students. J. Venom. Anim. Toxins. Incl. Trop. Dis. 21:1. doi: 10.1186/ 1678-9199-21-1

Wang, D. W., Liu, Y., Jiang, T. T., Zhang, G. X., Yuan, G. M., He, J. B., et al. (2016). Seroprevalence and genotypes of Toxoplasma gondii isolated from pigs intended for human consumption in Liaoning province, northeastern China. Parasit. Vectors 9:248. doi: 10.1186/s13071-016-1525-2

Xiao, Y., Yin, J. G., Jiang, N., Xiang, M., Hao, L. L., Lu, H. J., et al. (2010). Seroepidemiology of human Toxoplasma gondii infection in China. BMC. Infect. Dis. 10:4. doi: 10.1186/1471-2334-10-4

Yang, N., Mu, M. Y., Yuan, G. M., Zhang, G. X., Li, H. K., and He, J. B. (2013). Seroprevalence of Toxoplasma gondii in slaughtered horses and donkeys in Liaoning province, northeastern China. Parasit. Vectors 6:140. doi: 10.1186/ 1756-3305-6-140

Zemene, E., Yewhalaw, D., Abera, S., Belay, T., Samuel, A., and Zeynudin, A. (2012). Seroprevalence of Toxoplasma gondii and associated risk factors among pregnant women in Jimma town, Southwestern Ethiopia. BMC. Infect. Dis. 12:337. doi: 10.1186/1471-2334-12-337

Zhou, P., Chen, Z., Li, H. L., Zheng, H., He, S., Lin, R. Q., et al. (2011). Toxoplasma gondii infection in humans in China. Parasit. Vectors 4:165.

Conflict of Interest Statement: The authors declare that the research was conducted in the absence of any commercial or financial relationships that could be construed as a potential conflict of interest.

Copyright (c) 2017 Yang, Wang, Xing, Li, Li, Wu, Sang, Feng, Jiang and Chen. This is an open-access article distributed under the terms of the Creative Commons Attribution License (CC BY). The use, distribution or reproduction in other forums is permitted, provided the original author(s) or licensor are credited and that the original publication in this journal is cited, in accordance with accepted academic practice. No use, distribution or reproduction is permitted which does not comply with these terms. 